\title{
BOUNDARY LAYERS OF ARBITRARY DIMENSION FOR A SINGULARLY PERTURBED NEUMANN PROBLEM
}

\author{
Andrea Malchiodi* ${ }^{*}$ Marcelo Montenegro ${ }^{\dagger}$
}

This note is dedicated to the memory of Hebe Biagioni.

\begin{abstract}
We study the concentration phenomena for the equation $-\varepsilon^{2} \Delta u+u=$ $u^{p}$ in a smooth bounded domain $\Omega \subseteq \mathbb{R}^{n}$ and with Neumann boundary conditions. The exponent $p$ is greater or equal than 1 and the parameter $\varepsilon$ is converging to zero. For a suitable sequence $\varepsilon_{j} \rightarrow 0$ we prove existence of positive solutions $u_{j}$ concentrating at the whole boundary of $\Omega$ or at some of its components.
\end{abstract}

\section{Introduction}

This note features some recent results from [35] and [36]. We are concerned with concentration phenomena for solutions of the singularly-perturbed elliptic problem

$$
\begin{cases}-\varepsilon^{2} \Delta u+u=u^{p} & \text { in } \Omega, \\ \frac{\partial u}{\partial \nu}=0 & \text { on } \partial \Omega, \\ u>0 & \text { in } \Omega,\end{cases}
$$

where $\Omega \subseteq \mathbb{R}^{n}, n \geq 2$, is a smooth bounded domain, $\nu$ is the interior unit normal vector to $\partial \Omega, p>1$, and $\varepsilon$ is a small positive parameter. Our aim is to show the existence of a sequence $\varepsilon_{j} \rightarrow 0$ for which $\left(P_{\varepsilon_{j}}\right)$ admits solutions concentrating at $\partial \Omega$ or at some of its components.

Key Words: Singularly Perturbed Elliptic Problems, Local Inversion, Fourier Analysis.

AMS subject classification: 35B25, 35B34, 35J20, 35J60.

*Thanks UNICAMP for the hospitality.

†Supported by FAPESP 04/07035 and CNPq-478896/2003-4. 
Problem $\left(P_{\varepsilon}\right)$ arises in the study of pattern-formation for a class of reactiondiffusion equations. For example, in 1972 Gierer and Meinhardt proposed the following system

$$
\begin{cases}\mathcal{U}_{t}=d_{1} \Delta \mathcal{U}-\mathcal{U}+\frac{\mathcal{U}^{p}}{\mathcal{V}^{q}} & \text { in } \Omega \times(0,+\infty) \\ \mathcal{V}_{t}=d_{2} \Delta \mathcal{V}-\mathcal{V}+\frac{\mathcal{U}^{r}}{\mathcal{V}^{s}} & \text { in } \Omega \times(0,+\infty) \\ \frac{\partial \mathcal{U}}{\partial \nu}=\frac{\partial \mathcal{V}}{\partial \nu}=0 & \text { on } \partial \Omega \times(0,+\infty)\end{cases}
$$

to describe a biological experiment with hydra, see [22]. Here the functions $\mathcal{U}, \mathcal{V}$ represent the densities of a slowly-diffusing chemical activator and of a fast-diffusing inhibitor respectively. Therefore it is reasonable to assume $d_{1} \ll$ $1 \ll d_{2}$. The numbers $p, q, r, s$ are non-negative and satisfy the inequalities

$$
0<\frac{p-1}{q}<\frac{r}{s+1}
$$

By the so-called Turing's instability [46], system (GM) may exhibit non-trivial steady states when the coefficients $d_{1}$ and $d_{2}$ are very different. In the limit $d_{2} \rightarrow+\infty$, such states can be described by solutions of $\left(P_{\varepsilon}\right)$, see the survey [38] for details.

In the case $p<\frac{n+2}{n-2}$, problem $\left(P_{\varepsilon}\right)$ admits solutions, called spike-layers, concentrating at one or multiple points of $\bar{\Omega}$. It was first established in [32], [39], [40] that least-energy (mountain-pass) solutions of $\left(P_{\varepsilon}\right)$ are spike-layers which possess a local maximum point $Q_{\varepsilon}$ at $\partial \Omega$ such that $H\left(Q_{\varepsilon}\right) \rightarrow \max _{\partial \Omega} H$, where $H$ is the mean curvature of $\partial \Omega$.

Further studies on spike-layer solutions (for the Dirichlet problem as well) can be found in [17], [19], [24], [25], [26], [27], [30], [31], [47], [48]. Due to the work of the last ten-fifteen years, the phenomenon of concentration at points is now essentially understood, necessary and sufficient conditions for the location of boundary and interior spikes are available.

Roughly, a spike-layer solution $u_{\varepsilon}$ scales in the following way

$$
u_{\varepsilon}(x) \sim w_{0}(x / \varepsilon)
$$


More precisely, if $Q_{\varepsilon}$ is a local maximum of $u_{\varepsilon}$, then there holds

$$
u_{\varepsilon}\left(Q_{\varepsilon}+\varepsilon \cdot\right) \rightarrow w_{0}(\cdot) \quad \text { in } C_{l o c}^{2}\left(\mathbb{R}^{n}\right) \quad\left(\text { resp. in } C_{l o c}^{2}\left(\overline{\mathbb{R}_{+}^{n}}\right)\right),
$$

where $\mathbb{R}_{+}^{n}$ denotes the half-space $\left\{x_{n}>0\right\}$ and where $w_{0}$ is the unique positive radial solution of

$$
\begin{cases}-\Delta w_{0}+w_{0}=w_{0}^{p} & \text { in } \mathbb{R}^{n} \quad\left(\text { resp. in } \mathbb{R}_{+}^{n}\right) \\ w_{0}(x) \rightarrow 0 & \text { as }|x| \rightarrow+\infty .\end{cases}
$$

The limit domain is $\mathbb{R}^{n}$ or $\mathbb{R}_{+}^{n}$ if $Q_{\varepsilon}$ lies in the interior of $\Omega$ or on $\partial \Omega$ respectively.

Spike-layer solutions are produced using minimax methods, gluing techniques and, under suitable non-degeneracy conditions, by finite-dimensional reductions. Very recent results deal with the full system $(G M)$, see for example [20], [28], [41]. Similar techniques have been used for the study of the Nonlinear Schrödinger Equation (NLS) in the semiclassical limit, or other singularlyperturbed problems, see e.g. [2], [3], [7] and references therein.

It has been conjectured for some time that problem $\left(P_{\varepsilon}\right)$ should generically admit solutions concentrating on $k$-dimensional sets, for $k=1, \ldots, n-1$. The case $k=n$ is excluded since $\left(P_{\varepsilon}\right)$ is not expected to exhibit phase transitions.

In presence of symmetry some results have been proved in $[4,5,6,8,10,18]$ for problem $\left(P_{\varepsilon}\right)$, the Dirichlet problem, and the semiclassical NLS. See Remark 1.3 below for more precise comments.

In the general case, the above conjecture was first proved in [35] for the case $n=2$ and $k=1$, under the technical assumption $p \geq 2$. In the present note we settle the case $k=n-1$ for all $n$, assuming only the condition $p>1$. Hence, besides allowing $p \in(1,2)$, we can also deal with the supercritical case. This is mainly allowed by the fact that the limit profile of our solutions solves a one-dimensional problem (see the comments below), for which there is no restriction on $p$. Note that, by the results of [14], in the case of spike-layers (for the stationary NLS equation) the condition $p<\frac{n+2}{n-2}$ is also necessary for concentration. 
The solutions we obtain are boundary-layers and scale qualitatively in the following way, as $\varepsilon$ tends to zero

$$
u_{\varepsilon}\left(x^{\prime}, x_{n}\right) \sim w_{0}\left(x_{n} / \varepsilon\right) ; \quad x^{\prime} \in \mathbb{R}^{n-1}, x_{n} \in \mathbb{R}_{+},
$$

where now $w_{0}$ is the solution of the problem

$$
\begin{cases}-w_{0}^{\prime \prime}+w_{0}=w_{0}^{p} & \text { in } \mathbb{R}_{+} ; \\ w_{0}(x) \rightarrow 0 & \text { as } x \rightarrow+\infty \\ w_{0}^{\prime}(0)=0 & \end{cases}
$$

The result we are going to describe is the following.

Theorem 1.1 Let $\Omega \subseteq \mathbb{R}^{n}$ be a smooth bounded domain, and let $p>1$. Then there exists a sequence $\varepsilon_{j} \rightarrow 0$ and a sequence of solutions $u_{\varepsilon_{j}}$ of $\left(P_{\varepsilon_{j}}\right)$ with the following properties

(i) $u_{\varepsilon_{j}}$ concentrates around $\partial \Omega$ as $j \rightarrow+\infty$, namely for every $r>0$ one has $\int_{\Omega^{r}}\left(\varepsilon_{j}^{2}\left|\nabla u_{\varepsilon_{j}}\right|^{2}+u_{\varepsilon_{j}}^{2}\right) \rightarrow 0$ as $j \rightarrow+\infty$, where $\Omega^{r}=\{x \in \Omega$ : $\operatorname{dist}(x, \partial \Omega) \geq r\}$

(ii) if $x_{0} \in \partial \Omega$ and if $\nu_{0}$ denotes the interior unit normal to $\partial \Omega$ at $x_{0}$, then for every $k \in \mathbb{N}$ there holds

$$
u_{\varepsilon_{j}}\left(\varepsilon_{j}\left(x-x_{0}\right)\right) \rightarrow w_{0}\left(x \cdot \nu_{0}\right) \quad \text { in } C_{l o c}^{k}\left(V_{0}\right)
$$

where $V_{0}=\left\{x \in \mathbb{R}^{N}: x \cdot \nu_{0}>0\right\}$, and where $w_{0}$ is the solution of (1).

Using the scaling $u(x) \mapsto u(\varepsilon x)$, problem $\left(P_{\varepsilon}\right)$ is transformed into

$$
\begin{cases}-\Delta u+u=u^{p} & \text { in } \Omega_{\varepsilon} \\ \frac{\partial u}{\partial \nu}=0 & \text { on } \partial \Omega_{\varepsilon}, \\ u>0 & \text { in } \Omega_{\varepsilon}\end{cases}
$$

where $\Omega_{\varepsilon}=\frac{1}{\varepsilon} \Omega$. For $p \leq \frac{n+2}{n-2}$, solutions of $\left(\tilde{P}_{\varepsilon}\right)$ can be found as critical points of the functional $I_{\varepsilon}: H^{1}\left(\Omega_{\varepsilon}\right) \rightarrow \mathbb{R}$ defined by

$$
I_{\varepsilon}(u)=\frac{1}{2} \int_{\Omega_{\varepsilon}}\left(|\nabla u|^{2}+u^{2}\right)-\frac{1}{p+1} \int_{\Omega_{\varepsilon}}|u|^{p+1} ; \quad u \in H^{1}\left(\Omega_{\varepsilon}\right),
$$


where the space $H^{1}\left(\Omega_{\varepsilon}\right)$ is equipped with its standard norm $\|u\|^{2}=$ $\int_{\Omega_{\varepsilon}}\left(|\nabla u|^{2}+u^{2}\right)$. In the following, we use the notation $u_{\varepsilon}$ for both the solutions of $\left(P_{\varepsilon}\right)$ and $\left(\tilde{P}_{\varepsilon}\right)$.

The phenomenon of concentration at curves or manifolds for $\left(\tilde{P}_{\varepsilon}\right)$ is very different from that of concentration at points. Essentially, the difficulty here is that the energy of the solutions is very large (of order $\varepsilon^{1-n}$ ), and so is the Morse Index (also of order $\varepsilon^{1-n}$ ). The first assertion can be deduced by a simple dimensional argument, while the second is due to a loss of coercivity in the nonscaling direction of $u_{\varepsilon}$. For an heuristic explanation of this fact, we refer to the introduction of [35], see also Remark 2.14 below. Note also that we can prove existence just along a sequence $\varepsilon_{j}$. This seems to be a general property of this phenomenon, removable only in special cases, see Remark 1.2.

In the case of spike-layers, both the energy and the Morse index stays bounded as $\varepsilon$ goes to zero. Viceversa, by the results in [16], if the Morse index of a family of solutions stays bounded as $\varepsilon \rightarrow 0$, these solutions must concentrate at a finite number of points.

The proof of Theorem 1.1 is based on a local inversion argument. The main difficulty is that, since the Morse index of the solutions is changing with $\varepsilon$, the linearized operator $I_{\varepsilon}^{\prime \prime}\left(u_{\varepsilon}\right)$ will not be invertible for all the values of $\varepsilon$ in an interval $\left(0, \varepsilon_{0}\right)$. We get invertibility only along a sequence $\varepsilon_{j}$, and the norm of the inverse operator blows-up at the rate $\varepsilon_{j}^{n-1}$, see Proposition 2.12. This produces a resonance phenomenon which can be described as follows.

Let $\gamma \in(0,1)$, and let $\nu$ denote the inner unit normal to $\partial \Omega_{\varepsilon}$. Consider the neighborhood $\Sigma_{\varepsilon}$ of $\partial \Omega_{\varepsilon}$ defined as

$$
\Sigma_{\varepsilon}=\left\{x^{\prime}+x_{n} \nu: x^{\prime} \in \partial \Omega_{\varepsilon}, x_{n} \in\left(0, \varepsilon^{-\gamma}\right)\right\}
$$

Let $\left\{\phi_{l}\right\}_{l}$ denote the eigenfunctions of $-\Delta_{\hat{g}}$, where $\Delta_{\hat{g}}$ is the Laplace-Beltrami operator on $\partial \Omega$, and let $\left\{\lambda_{l}\right\}_{l}$ be the corresponding (non-negative) eigenvalues.

Let $z_{\sigma}$ be an eigenfunction of $I_{\varepsilon}^{\prime \prime}\left(u_{\varepsilon}\right)$ with eigenvalue $\sigma$. Then we can de- 
compose $z_{\sigma}$ in Fourier series (in the variable $x^{\prime}$ ) in the following way

$$
z_{\sigma}\left(x^{\prime}, x_{n}\right)=\sum_{l=0}^{\infty} \phi_{l}\left(\varepsilon x^{\prime}\right) z_{\sigma, l}\left(x_{n}\right) ; \quad x^{\prime} \in \partial \Omega_{\varepsilon}, x_{n} \in\left(0, \varepsilon^{-\gamma}\right) .
$$

If the eigenvalue $\sigma$ is close to zero, it turns out that all the modes $\phi_{l} z_{\sigma, l}$ are negligible, except those for which $\lambda_{l} \sim \varepsilon^{-2}$, see also formula (3). This is stated rigorously in Proposition 2.9. It follows that, qualitatively, the resonant eigenvalues of $I_{\varepsilon}^{\prime \prime}\left(u_{\varepsilon}\right)$ have eigenfunctions with more and more oscillations along $\partial \Omega_{\varepsilon}$ as $\varepsilon$ tends to zero.

We describe below the general procedure employed here to attack the problem. The proof of our main result is based on the Contraction Mapping Theorem, once two preliminary steps are accomplished.

Step 1: finding an approximate solution. Given an arbitrary positive number $\theta$, we are able to find an approximate solution $u_{k, \varepsilon}$ of $\left(\tilde{P}_{\varepsilon}\right)$ for which $\left\|I_{\varepsilon}^{\prime}\left(u_{k, \varepsilon}\right)\right\|=O\left(\varepsilon^{\theta}\right)$. The function $u_{k, \varepsilon}$ is constructed essentially by power series in $\varepsilon$ with $k$ iterations, where the number $k$ depends on $n, p$ and $\theta$. Thus $u_{k, \varepsilon}$ has roughly the following form

$$
\begin{aligned}
& u_{k, \varepsilon}\left(x^{\prime}, x_{n}\right)=w_{0}\left(x_{n}\right)+\varepsilon \tilde{w}_{1}\left(\varepsilon x^{\prime}, x_{n}\right)+\ldots \varepsilon^{k} \tilde{w}_{k}\left(\varepsilon x^{\prime}, x_{n}\right), \\
& x^{\prime} \in \partial \Omega_{\varepsilon}, x_{n} \in J_{\varepsilon}=\left[0, \varepsilon^{-\gamma}\right] .
\end{aligned}
$$

Here $\tilde{w}_{1}, \ldots, \tilde{w}_{k}$ are smooth functions on $\partial \Omega \times J_{\varepsilon}$, which are defined inductively in their index. Basically, each function $\tilde{w}_{i}$ is obtained by an inversion argument from $\tilde{w}_{0}, \ldots, \tilde{w}_{i-1}$ and the geometry of $\Omega$. Despite the resonance phenomena of the operator $I_{\varepsilon}^{\prime \prime}$, here we can perform this inversion because we are assuming a smooth dependence on the variable $\varepsilon x^{\prime}$. In some sense, since resonance occurs only at high frequencies, see the comments above, smooth functions on $\partial \Omega$ (scaled to $\Omega_{\varepsilon}$ ) are not affected by this phenomenon since their Fourier modes are mainly low-frequency ones. The rigorous derivation of $u_{k, \varepsilon}$ is performed in Subsection 2.2. The smoothness of $\Omega$ is essential to construct $u_{k, \varepsilon}$.

In [35], for $n=2$, we were able to satisfy the above requirement only for $\theta=\frac{3}{2}$. To deal with a general $n$ we need a better approximation since both the 
energy of the solutions and the norm of the inverse operator grow faster when the dimension $n$ is larger.

Step 2: inverting the linearized operator $I_{\varepsilon}^{\prime \prime}\left(u_{k, \varepsilon}\right)$. This is a rather delicate issue since, as we remarked before, the linearized operator is not invertible for all the values of $\varepsilon$. Qualitatively, using the Weyl's asymptotic formula, one finds that the $l$-th eigenvalue $\sigma_{l}$ of $I_{\varepsilon}^{\prime \prime}\left(u_{k, \varepsilon}\right)$ is given by

$$
\sigma_{l} \sim-1+\varepsilon^{2} l^{\frac{2}{n-1}}
$$

see Subsection 2.3 for details. It follows that $\sigma_{l} \sim 0$ for $l \sim \varepsilon^{1-n}$, and that the average distance between two consecutive eigenvalues close to zero is of order $\varepsilon^{n-1}$. We show indeed that along a sequence $\varepsilon_{j}$ the spectrum of the linearized operator stays away from zero of order $\varepsilon_{j}^{n-1}$, and hence we get an inverse operator with norm proportional to $\varepsilon_{j}^{-(n-1)}$. The way to prove this fact is based on a first rough comparison of the eigenvalues with those (essentially known, see Proposition 2.5) of a model problem, obtaining an estimate of the Morse index of the solutions. Then, Kato's Theorem allows us to choose the values of $\varepsilon$ appropriately and to invert the linear operator along a sequence $\varepsilon_{j}$. Notice that Kato's Theorem requires some information not only on the eigenvalues, but also on the eigenfunctions, see Subsection 2.1.

Final step: the contraction argument. If the operator $I_{\varepsilon}^{\prime \prime}\left(u_{k, \varepsilon}\right)$ is invertible, a function $u_{\varepsilon}$ of the form $u_{\varepsilon}=u_{k, \varepsilon}+w$ is a solution of $\left(\tilde{P}_{\varepsilon}\right)$ if and only if $w$ is a fixed point of the operator $F_{\varepsilon}$, where $F_{\varepsilon}$ is defined as

$$
F_{\varepsilon}(w)=-I_{\varepsilon}^{\prime \prime}\left(u_{k, \varepsilon}\right)^{-1}\left[I_{\varepsilon}^{\prime}\left(u_{k, \varepsilon}\right)+G(w)\right] .
$$

Here $G(w)$ is a superlinear term satisfying $\|G(w)\| \leq O(\|w\|)^{\min \{2, p\}}$. Since along the sequence $\varepsilon_{j}$ the norm of the operator $I_{\varepsilon}^{\prime \prime}\left(u_{k, \varepsilon}\right)^{-1}$ is of order $\varepsilon^{-n}$, we need to choose $\theta$ to be sufficiently large (see Step 1), depending also on $p$, in order to get a contraction. 
A further difficulty in dealing with the case $n \geq 3$ is that the exponent $p$ could also be supercritical. This case is tackled with a truncation argument, proving a priori estimates in $L^{\infty}$. These are based on a combination of norm estimates, obtained using Step 2, and elliptic regularity theory.

Remark 1.2 We do not expect that in general concentration at manifolds of dimension $k$, with $k \geq 1$, occurs along a continuous range of $\varepsilon$. This is possible (and verified) instead in very particular situations, under some symmetry assumptions. In this case the resonance phenomenon can be ruled out working in spaces of symmetric functions. However, since the Morse index of the solutions is still changing with $\varepsilon$, bifurcation of non-symmetric solutions occurs from the branch of the symmetric ones, see [4], [15], [45].

For the general case it is reasonable to expect imperfect bifurcation, namely that the set $\left(\varepsilon, u_{\varepsilon}\right) \subseteq \mathbb{R} \times H^{1}(\Omega)$, where $u_{\varepsilon}$ is a boundary-layer, looks like an hyperbola when the Morse index of $u_{k, \varepsilon}$ is changing.

Similar resonance phenomena were observed in [34], [44] for a class of ordinary differential equations. We believe that a similar picture should take place also in this case.

Remark 1.3 Under symmetry assumptions, it is possible to analyze different aspects of concentration at manifolds.

In [4], [8] it is considered the case of standing waves for the semiclassical Nonlinear Schrödinger Equation, and it is shown how the location of the concentration set arises as a combination of the effects of the potential and the volume of the support of the solutions (this phenomenon is absent in the case of concentration at points). In particular in [4] necessary and sufficient conditions are given for the location of the concentration set.

In [8], [18] concentration on circles is produced considering solutions of the NLS with non-vanishing angular momentum, and the potential is now balancing volume and centrifugal forces.

In [5] it is analyzed the effect of the boundary of the domain. In particular, for problem $\left(P_{\varepsilon}\right)$ in a ball, it is shown that there exist solutions with a spherical 
crown of maxima at distance $\varepsilon|\log \varepsilon|$ from the boundary. The radial profile of these solutions is that of an interior spike, hence they are of different type with respect to those analyzed here.

Remark 1.4 (A) Concentration along curves or manifolds also occurs for other classes of equations, like Allen-Cahn, Ginzburg-Landau or Yang-Mills, see e.g. the recent papers [11], [12], [43].

The phenomenon is different from our case, since the resonance phenomena there (although present) do not affect so drastically the structure of the solutions. In fact, under generic non-degeneracy assumptions, concentration occurs without gaps in the scaling parameter.

The main reason for this difference is the following. For these equations the basic solution (transition layer, vortex, instanton) in the direction orthogonal to the manifold of concentration is, loosely speaking a minimum. In our case, instead, the function $w_{0}$ is a mountain-pass, giving rise to a negative eigenvalue for the 1-dimensional problem, see Proposition 2.1. As a consequence the eigenvalues of the linearized operator in $n$ dimensions grow from a negative number and eventually cross zero, as described in formula (3).

(B) Problems of finding constant mean curvature surfaces shrinking at a curve or along a manifold present similar features, see [33] and [37].

The outline of this note is the following. In Subsection 2.1 we collect some preliminary facts and we study a family of auxiliary one-dimensional problems, proving some continuity and monotonicity properties. Subsection 2.2 is devoted to the construction of the approximate solution $u_{k, \varepsilon}$. In Subsection 2.3 we give a characterization of the eigenfunctions and the eigenvalues of the operator $T_{\Sigma_{\varepsilon}}$, which basically coincide with those of $I_{\varepsilon}^{\prime \prime}\left(u_{k, \varepsilon}\right)$, see Proposition 2.13. In Subsection 2.4 we prove Theorem 1.1.

\section{Idea of the proof of Theorem 1.1}

In this section we give a sketch of the proof of theorem 1.1. We provide complete proofs of a couple of results, referring to [35] and [36] for those which we do not 
report here.

\subsection{Notation and preliminary facts}

In this subsection we introduce some notation which will be frequently used later on, and we present the linear theory of some auxiliary one-dimensional problems.

Throughout this note, $C$ denotes a large positive constant. For convenience, we allow $C$ to vary among formulas (also within the same line) and to assume larger and larger values. The number $\gamma$ will be fixed in Subsection 2.2. We often work in the functional space $H^{1}\left(\Omega_{\varepsilon}\right)$ endowed with its natural norm, which we denote simply by $\|\cdot\|$, without any subscript. Solutions of $\left(\tilde{P}_{\varepsilon}\right)$ for $p \leq \frac{n+2}{n-2}$ are found as critical points of the functional $I_{\varepsilon}: H^{1}\left(\Omega_{\varepsilon}\right) \rightarrow \mathbb{R}$ defined in (2).

Consider the problem

$$
\begin{cases}-u^{\prime \prime}+u=u^{p} & \text { in } \mathbb{R}_{+} \\ u>0 & \text { in } \mathbb{R}_{+} \\ u^{\prime}(0)=0, & \end{cases}
$$

with $p>1$. It is well-known, see e.g. [21], that problem (4) possesses a solution $w_{0}$ which satisfies the properties

$$
\begin{cases}w_{0}^{\prime}(r)<0, & \text { for all } r>0, \\ \lim _{r \rightarrow \infty} e^{r} w_{0}(r)=\alpha_{p}>0, & \lim _{r \rightarrow \infty} \frac{w_{0}^{\prime}(r)}{w_{0}(r)}=-1,\end{cases}
$$

where $\alpha_{p}$ is a positive constant depending only on $p$. Using some ODE analysis, one can see that all the solutions of $(4)$ in $H^{1}\left(\mathbb{R}_{+}\right)$coincide with $w_{0}$.

Solutions of problem (4) in the class $H^{1}\left(\mathbb{R}_{+}\right)$are critical points of the functional $\bar{I}$ defined as

$$
\bar{I}(u)=\frac{1}{2} \int_{\mathbb{R}_{+}}\left(\left(u^{\prime}\right)^{2}+u^{2}\right)-\frac{1}{p+1} \int_{\mathbb{R}_{+}}|u|^{p+1}, \quad u \in H^{1}\left(\mathbb{R}_{+}\right) .
$$

We have the following non-degeneracy result, see e.g. [21], [42].

Proposition 2.1 The function $w_{0}$ is a non-degenerate critical point of $\bar{I}$. Precisely, there exists a positive constant $C$ such that

$$
\begin{gathered}
\bar{I}^{\prime \prime}\left(w_{0}\right)\left[w_{0}, w_{0}\right] \leq-C^{-1}\left\|w_{0}\right\|_{0}^{2} ; \quad \bar{I}^{\prime \prime}\left(w_{0}\right)[v, v] \geq C^{-1}\|v\|_{0}^{2}, \\
\text { for all } v \in H^{1}\left(\mathbb{R}_{+}\right), v \perp w_{0},
\end{gathered}
$$


where $\|\cdot\|_{0}$ denotes the standard norm of $H^{1}\left(\mathbb{R}_{+}\right)$. As a consequence, we have $\mu<0$ and $\tau>0$, where $\mu$ and $\tau$ are respectively the first and the second eigenvalues of $\bar{I}^{\prime \prime}\left(w_{0}\right)$. Furthermore the eigenvalue $\mu$ is simple.

Our next goal is to characterize the eigenvalues $\sigma$ (in particular the first two) of the following problem

$$
\left\{\begin{array}{l}
(1-\sigma)\left(-u^{\prime \prime}+(1+\alpha) u\right)=p w_{0}^{p-1} u, \quad \text { in } \mathbb{R}_{+} \\
u^{\prime}(0)=0
\end{array}\right.
$$

where $\alpha$ is a positive parameter. Equation (7) arises in the study of some linear partial differential equation after performing a Fourier decomposition, see equation (15) below.

In order to study the eigenvalues of (7), it is convenient to introduce the following norm $\|\cdot\|_{\alpha}$ on $H^{1}\left(\mathbb{R}_{+}\right)$

$$
\|u\|_{\alpha}^{2}=\int_{\mathbb{R}_{+}}\left(\left(u^{\prime}\right)^{2}+(1+\alpha) u^{2}\right) ; \quad u \in H^{1}\left(\mathbb{R}_{+}\right) .
$$

Let $H_{\alpha}$ be the Hilbert space consisting of $H^{1}\left(\mathbb{R}_{+}\right)$and endowed with this norm. We denote by $(,)_{\alpha}$ the corresponding scalar product. We also define by duality the operator $T_{\alpha}: H_{\alpha} \rightarrow H_{\alpha}$ in the following way

$$
\left(T_{\alpha} u, v\right)_{\alpha}=\int_{\mathbb{R}_{+}}\left(u^{\prime} v^{\prime}+(1+\alpha) u v-p w_{0}^{p-1} u v\right) ; \quad u, v \in H_{\alpha} .
$$

Then equation (7) can be written in the abstract form

$$
T_{\alpha} u=\sigma u ; \quad u \in H_{\alpha}
$$

Note that when $\alpha=0, T_{\alpha}$ coincides with $\bar{I}^{\prime \prime}\left(w_{0}\right)$. We have the following result.

Proposition 2.2 Let $\mu_{\alpha}$ and $\tau_{\alpha}$ denote respectively the first and the second eigenvalues of $T_{\alpha}$. Then $\mu_{\alpha}$ is simple and the following properties hold true

(i) $\alpha \mapsto \mu_{\alpha}$ is smooth and strictly increasing;

(ii) $\alpha \mapsto \tau_{\alpha}$ is non-decreasing. 
The eigenfunction $v_{\alpha}$ of $T_{\alpha}$ corresponding to $\mu_{\alpha}$, normalized with $\left\|v_{\alpha}\right\|_{\alpha}=1$, can be chosen to be positive and strictly decreasing on $\mathbb{R}_{+}$. The map $\alpha \mapsto v_{\alpha}$ is smooth from $\mathbb{R}$ into $H^{1}\left(\mathbb{R}_{+}\right)$. Furthermore, $\mu_{\alpha}>0$ for $\alpha$ sufficiently large.

Given $\alpha>0$, we also define the function $\tilde{F}(\alpha)$ to be

$$
\tilde{F}(\alpha)=2 \alpha\left(1-\mu_{\alpha}\right) \int_{\mathbb{R}_{+}} v_{\alpha}^{2}>0
$$

Note that, by the smoothness of $\alpha \mapsto v_{\alpha}$, also the function $\alpha \mapsto \tilde{F}(\alpha)$ is smooth.

We also need to consider a variant of the eigenvalue problem $(7)$. For $\gamma \in(0,1)$, let $J_{\varepsilon}$ denote the interval

$$
J_{\varepsilon}=\left[0, \varepsilon^{-\gamma}\right]
$$

and let

$$
H_{\varepsilon}^{1}=\left\{u \in H^{1}\left(J_{\varepsilon}\right): u\left(\varepsilon^{-\gamma}\right)=0\right\}
$$

We let $H_{\alpha, \varepsilon}$ denote the space $H_{\varepsilon}^{1}$ endowed with the norm

$$
\|u\|_{\alpha, \varepsilon}^{2}=\int_{J_{\varepsilon}}\left(\left(u^{\prime}\right)^{2}+(1+\alpha) u^{2}\right) ; \quad u \in H_{\varepsilon}^{1}
$$

and $(,)_{\alpha, \varepsilon}$ the corresponding scalar product. Similarly, we define $T_{\alpha, \varepsilon}$ by

$$
\left(T_{\alpha, \varepsilon} u, v\right)_{\alpha, \varepsilon}=\int_{J_{\varepsilon}}\left(u^{\prime} v^{\prime}+(1+\alpha) u v-p w_{0}^{p-1} u v\right) ; \quad u, v \in H_{\alpha, \varepsilon}
$$

The operator $T_{\alpha, \varepsilon}$ satisfies properties analogous to $T_{\alpha}$. We list them in the next Proposition, which also gives a comparison between the first eigenvalues and eigenfunctions of $T_{\alpha}$ and $T_{\alpha, \varepsilon}$.

Proposition 2.3 Let $\mu_{\alpha, \varepsilon}$ and $\tau_{\alpha, \varepsilon}$ denote respectively the first and the second eigenvalues of $T_{\alpha, \varepsilon}$. Then $\mu_{\alpha, \varepsilon}$ is simple and the following properties hold true

(i) $\alpha \mapsto \mu_{\alpha, \varepsilon}$ is smooth and strictly increasing;

(ii) $\alpha \mapsto \tau_{\alpha, \varepsilon}$ is non-decreasing. 
The eigenfunction $v_{\alpha, \varepsilon}$ of $T_{\alpha, \varepsilon}$ corresponding to $\mu_{\alpha, \varepsilon}$, normalized with $\left\|v_{\alpha, \varepsilon}\right\|_{\alpha, \varepsilon}=$ 1 , can be chosen to be positive and strictly decreasing on $\mathbb{R}_{+}$. Moreover there exist a large constant $C$ and a small constant $\delta$ depending only on $p$ such that $\left|\mu_{\alpha}-\mu_{\alpha, \varepsilon}\right| \leq C e^{-\delta \varepsilon^{-\gamma}} ; \quad\left\|v_{\alpha}-v_{\alpha, \varepsilon}\right\|_{H^{1}\left(\mathbb{R}_{+}\right)} \leq C \varepsilon^{-\frac{1}{2} \gamma} e^{-\delta \varepsilon^{-\gamma}}, \quad$ for $\mu_{\alpha} \in\left[-\frac{1}{4} \tau, \frac{1}{4} \tau\right]$ and for $\varepsilon$ small. The function $v_{\alpha, \varepsilon}$ in this formula has been set identically 0 outside $\left[0, \varepsilon^{-\gamma}\right]$.

Remark 2.4 Using the Courant-Fisher method, one can also prove that $\tau_{\alpha, \varepsilon} \geq$ $\tau_{\alpha}$ for all values of $\alpha$ and $\varepsilon$.

We use Fourier analysis in order to study equation $\left(\tilde{P}_{\varepsilon}\right)$. For $\gamma>0$, let us define the set $S_{\varepsilon}$ and the metric $g_{0}$ on $S_{\varepsilon}$ as

$$
S_{\varepsilon}=\partial \Omega_{\varepsilon} \times J_{\varepsilon}=\partial \Omega_{\varepsilon} \times\left[0, \varepsilon^{-\gamma}\right], \quad g_{0}=\bar{g}_{\varepsilon} \otimes(d t)^{2},
$$

where $\bar{g}_{\varepsilon}$ is the metric on $\partial \Omega_{\varepsilon}$ induced by $\mathbb{R}^{n}$. We also define the functional space

$$
H_{S_{\varepsilon}}=\left\{u \in H^{1}\left(S_{\varepsilon}\right): u\left(x^{\prime}, \varepsilon^{-\gamma}\right)=0 \text { for all } x^{\prime} \in \partial \Omega_{\varepsilon}\right\}
$$

endowed with the norm

$$
\|u\|_{H_{S_{\varepsilon}}}^{2}=\int_{S_{\varepsilon}}\left(\left|\nabla_{g_{0}} u\right|^{2}+u^{2}\right) ; \quad u \in H_{S_{\varepsilon}} .
$$

Let $\hat{g}$ be the metric on $\partial \Omega$ induced by that of $\mathbb{R}^{n}$, and let $\Delta_{\hat{g}}$ be the LaplaceBeltrami operator. Let $\left\{\lambda_{l}\right\}_{l}$ denote the eigenvalues of $-\Delta_{\hat{g}}$, counted in nondecreasing order and with their multiplicity. Let also $\left\{\phi_{l}\right\}_{l}$ denote the corresponding eigenfunctions.

Given $u \in H_{S_{\varepsilon}}$, we can decompose it in Fourier components (in the variable $\left.x^{\prime}\right)$ as follows

$$
u\left(x^{\prime}, x_{n}\right)=\sum_{l=0}^{\infty} \phi_{l}\left(\varepsilon x^{\prime}\right) u_{l}\left(x_{n}\right), \quad x^{\prime} \in \partial \Omega_{\varepsilon}, x_{n} \in J_{\varepsilon} .
$$

Using this decomposition, one finds

$$
\|u\|_{H_{S_{\varepsilon}}}^{2}=\frac{1}{\varepsilon^{n-1}} \sum_{l} \int_{J_{\varepsilon}}\left(\left(u_{l}^{\prime}\right)^{2}+\left(1+\varepsilon^{2} \lambda_{l}\right) u_{l}^{2}\right)=\frac{1}{\varepsilon^{n-1}} \sum_{l}\left\|u_{l}\right\|_{\varepsilon^{2} \mu_{l}}^{2}
$$


Writing for brevity $\|\cdot\|_{l, \varepsilon}$ instead of $\|\cdot\|_{\varepsilon^{2} \mu_{l}}$, the last equation becomes

$$
\|u\|_{H_{S_{\varepsilon}}}^{2}=\frac{1}{\varepsilon^{n-1}} \sum_{l}\left\|u_{l}\right\|_{l, \varepsilon}^{2} .
$$

This is how the norms $\|\cdot\|_{\alpha}$ introduced above enter in our study; in particular we will choose $\alpha$ belonging to the discrete set $\left\{\varepsilon^{2} \lambda_{l}\right\}_{l}$.

We are also interested in an eigenvalue problem of the form

$$
T_{S_{\varepsilon}} u=\sigma u ; \quad u \in H_{S_{\varepsilon}},
$$

where $T_{S_{\varepsilon}}$ is defined by

$$
\left(T_{S_{\varepsilon}} u, v\right)=\int_{S_{\varepsilon}}\left(\nabla_{g_{0}} u \cdot \nabla_{g_{0}} v+u v-p w_{0}^{p-1} u v\right) d V_{g_{0}}, \quad u, v \in H_{S_{\varepsilon}} .
$$

Writing $u$ as in (12), equation (14) is equivalent to

$$
\left\{\begin{array}{l}
(1-\sigma)\left(-u_{l}^{\prime \prime}+\left(1+\varepsilon^{2} \lambda_{l}\right) u_{l}\right)=p w_{0}^{p-1} u_{l} \quad \text { in } J_{\varepsilon}, \quad \text { for all } l . \\
u_{l}^{\prime}(0)=0
\end{array}\right.
$$

Since $T_{S_{\varepsilon}}$ represents a model for the study of $I_{\varepsilon}^{\prime \prime}\left(u_{k, \varepsilon}\right)$, it was essential for us to perform a spectral analysis of the operators $T_{\alpha}$.

The spectrum of $T_{S_{\varepsilon}}$ is characterized in the next proposition. We recall the definition of the (positive) number $\tau$ in Proposition 2.1.

Proposition 2.5 Let $\sigma<\frac{\tau}{4}$ be an eigenvalue of $T_{S_{\varepsilon}}$. Then $\sigma=\mu_{l, \varepsilon}$ for some index $l$. The corresponding eigenfunctions $u$ are of the form

$$
u\left(x^{\prime}, x_{n}\right)=\sum_{\left\{l: \mu_{l, \varepsilon}=\sigma\right\}} \alpha_{l} \phi_{l}\left(\varepsilon x^{\prime}\right) v_{l, \varepsilon}\left(x_{n}\right), \quad x^{\prime} \in \partial \Omega_{\varepsilon}, x_{n} \in J_{\varepsilon},
$$

where $\left\{\alpha_{l}\right\}_{l}$ are arbitrary constants. Viceversa, every function of the form (16) is an eigenfunction of $T_{S_{\varepsilon}}$ with eigenvalue $\sigma$. In particular the eigenvalues of $T_{S_{\varepsilon}}$ which are smaller than $\tau$ coincide with the numbers $\left\{\mu_{l, \varepsilon}\right\}_{l}$ which are smaller than $\tau$.

Proof. Let $u$ be an eigenfunction of $T_{S_{\varepsilon}}$ with eigenvalue $\sigma$. Then we can write

$$
u\left(x^{\prime}, x_{n}\right)=\sum_{l}\left(\alpha_{l} v_{l, \varepsilon}\left(x_{n}\right)+\hat{v}_{l}\left(x_{n}\right)\right) \phi_{l}\left(\varepsilon x^{\prime}\right)
$$


where $\alpha_{l}$ are real numbers, and where $\left(\hat{v}_{l}, v_{l, \varepsilon}\right)_{l, \varepsilon}=0$ for all $l$. We have

$$
\begin{aligned}
\sum_{l}\left(\alpha_{l} \mu_{l, \varepsilon} v_{l, \varepsilon}\left(x_{n}\right)+T_{l, \varepsilon} \hat{v}_{l}\left(x_{n}\right)\right) \phi_{l}\left(\varepsilon x^{\prime}\right) & =T_{S_{\varepsilon}} u=\sigma u \\
& =\sigma \sum_{l}\left(\alpha_{l} v_{l, \varepsilon}\left(x_{n}\right)+\hat{v}_{l}\left(x_{n}\right)\right) \phi_{l}\left(\varepsilon x^{\prime}\right) .
\end{aligned}
$$

By the uniqueness of the Fourier decomposition, the last equation implies

$$
\mu_{l, \varepsilon}=\sigma \quad \text { if } \quad \alpha_{l} \neq 0 ; \quad \text { and } \quad T_{l, \varepsilon} \hat{\varepsilon}_{l}=\sigma \hat{v}_{l} \quad \text { for all } l .
$$

Proposition $2.3 \mathrm{ii}$ ) and Remark 2.4 yield

$$
\tau_{\alpha, \varepsilon} \geq \tau_{\alpha} \geq \tau
$$

which means that $T_{\alpha, \varepsilon} \geq \tau I d$ on the subspace of $H_{\alpha, \varepsilon}$ orthogonal to $v_{\alpha, \varepsilon}$. Hence the second equation in (17) and the fact that $\sigma<\frac{\tau}{4}$ imply $\hat{v}_{l}=0$ for all $l$. Moreover, since $\lambda_{l} \rightarrow \mu_{l, \varepsilon}$ is a monotone function, the first equation in (18) shows that all the indices $l$ for which $\alpha_{l} \neq 0$ correspond to the same value $\lambda_{l}$. This concludes the proof.

We finally recall the following theorem due to T. Kato, ([29], page 444) which is fundamental to us in order to obtain invertibility, see Proposition 2.12.

Theorem 2.6 Let $T(\chi)$ denote a differentiable family of operators from an Hilbert space $X$ into itself, where $\chi$ belongs to an interval containing 0 . Let $T(0)$ be a self-adjoint operator of the form Identity - compact and let $\sigma(0)=\sigma_{0} \neq 1$ be an eigenvalue of $T(0)$. Then the eigenvalue $\sigma(\chi)$ is differentiable at 0 with respect to $\chi$. The derivative of $\sigma$ is given by

$$
\frac{\partial \sigma}{\partial \chi}=\left\{\text { eigenvalues of } P_{\sigma_{0}} \circ \frac{\partial T}{\partial \chi}(0) \circ P_{\sigma_{0}}\right\},
$$

where $P_{\sigma_{0}}: X \rightarrow X_{\sigma_{0}}$ denotes the projection onto the $\sigma_{0}$ eigenspace $X_{\sigma_{0}}$ of $T(0)$.

Remark 2.7 We note that, when perturbing the operator $T(0)$, the eigenvalue $\sigma_{0}$ can split in several ones. Hence in general $\sigma_{0}$ possesses a multivalued derivative. Anyway, since the operator T(0) is of the form Identity - compact, and since $\sigma_{0}$ is different from $1, \sigma_{0}$ is an isolated eigenvalue and the projection $P_{\sigma_{0}}$ has a finite dimensional range. Hence the splitting of $\sigma_{0}$ is finite. 


\subsection{An approximate solution}

In this subsection we construct approximate solutions $u_{k, \varepsilon}$ of $\left(\tilde{P}_{\varepsilon}\right)$ of arbitrary order in $\varepsilon$. Basically we expand a solution $u_{\varepsilon}$ of $\left(\tilde{P}_{\varepsilon}\right)$ in powers of $\varepsilon$ and then use suitable truncations.

Proposition 2.8 Consider the Euler functional $I_{\varepsilon}$ defined in (2) and associated to problem $\left(\tilde{P}_{\varepsilon}\right)$. Then for any $k \in \mathbb{N}$ there exists a function $u_{k, \varepsilon}: S_{\varepsilon} \rightarrow \mathbb{R}$ with the following properties

$$
\left\|I_{\varepsilon}^{\prime}\left(u_{k, \varepsilon}\right)\right\| \leq C_{k} \varepsilon^{k+1-\frac{n-1}{2}} ; \quad u_{k, \varepsilon} \geq 0 \quad \text { in } \Omega_{\varepsilon} \quad \frac{\partial u_{k, \varepsilon}}{\partial \nu}=0 \quad \text { on } \partial \Omega_{\varepsilon}
$$

where $C_{k}$ depends only on $\Omega, p$ and $k$. Moreover there holds

$$
\left\{\begin{array}{l}
\left\|\left(\nabla^{\prime}\right)^{(m)} u_{k, \varepsilon}\left(x^{\prime}, x_{n}\right)\right\|^{\prime} \leq C_{m} \varepsilon^{m} e^{-x_{n}} ; \\
\left\|\left(\nabla^{\prime}\right)^{(m)} \partial_{x_{n}} u_{k, \varepsilon}\left(x^{\prime}, x_{n}\right)\right\|^{\prime} \leq C_{m} \varepsilon^{m} e^{-x_{n}} ; \quad x^{\prime} \in \partial \Omega_{\varepsilon}, x_{n} \in J_{\varepsilon}, m=0,1, \ldots, \\
\left\|\left(\nabla^{\prime}\right)^{(m)} \partial_{x_{n}}^{2} u_{k, \varepsilon}\left(x^{\prime}, x_{n}\right)\right\|^{\prime} \leq C_{m} \varepsilon^{m} e^{-x_{n}} ;
\end{array}\right.
$$

where $\nabla^{\prime}$ and $\|\cdot\|^{\prime}$ denote the derivative and the norm taken with respect to the variable $x^{\prime}$ (freezing $x_{n}$ ), and $C_{m}$ is a constant depending only on $\Omega, p$ and $m$.

We parameterize the set $S_{\varepsilon}$, see equation (11), using coordinates $x^{\prime}$ on $\partial \Omega_{\varepsilon}$ and $x_{n}$ in $J_{\varepsilon}$. Let $\nu$ denote the unit inner normal to $\partial \Omega$, and define the map $\Gamma_{\varepsilon}: \partial \Omega_{\varepsilon} \times J_{\varepsilon} \rightarrow \mathbb{R}^{n}$ by

$$
\Gamma_{\varepsilon}\left(x^{\prime}, x_{n}\right)=x^{\prime}+x_{n} \nu\left(\varepsilon x^{\prime}\right)
$$

We let the upper-case indices $A, B, C, \ldots$ run from 1 to $n$, and the lower-case indices $i, j, k, \ldots$ run from 1 to $n-1$. Using some local coordinates $\left\{x_{i}\right\}_{i=1, \ldots, n-1}$ on $\partial \Omega_{\varepsilon}$, and letting $\varphi_{\varepsilon}$ be the corresponding immersion into $\mathbb{R}^{n}$, we have

$$
\left\{\begin{array}{lr}
\frac{\partial \Gamma_{\varepsilon}}{\partial x_{i}}\left(x^{\prime}, x_{n}\right)=\frac{\partial \varphi_{\varepsilon}}{\partial x_{i}}\left(x^{\prime}\right)+\varepsilon x_{n} \frac{\partial \nu}{\partial x_{i}}\left(\varepsilon x^{\prime}\right)=\frac{\partial \varphi_{\varepsilon}}{\partial x_{i}}\left(x^{\prime}\right)+\varepsilon x_{n} H_{i}^{j}\left(\varepsilon x^{\prime}\right) \frac{\partial \varphi_{\varepsilon}}{\partial x_{j}}\left(x^{\prime}\right), \\
\frac{\partial \Gamma_{\varepsilon}}{\partial x_{n}}\left(x^{\prime}, x_{n}\right)=\nu\left(\varepsilon x^{\prime}\right) & \text { for } i=1, \ldots, n-1 ;
\end{array}\right.
$$

where $\left\{H_{i}^{j}\right\}$ are the coefficients of the mean-curvature operator on $\partial \Omega$. Let also $\bar{g}_{i j}$ be the coefficients of the metric on $\partial \Omega_{\varepsilon}$ in the above coordinates $\left(x^{\prime}, x_{n}\right)$. 
Then, letting $g=g_{\varepsilon}$ denote the metric on $\Omega_{\varepsilon}$ induced by $\mathbb{R}^{n}$, we have

$$
g_{A B}=\left(\frac{\partial \Gamma_{\varepsilon}}{\partial x_{A}}, \frac{\partial \Gamma_{\varepsilon}}{\partial x_{B}}\right)=\left(\begin{array}{cc}
\left\{g_{i j}\right\} & 0 \\
0 & 1
\end{array}\right),
$$

where

$$
\begin{aligned}
g_{i j} & =\left(\frac{\partial \varphi_{\varepsilon}}{\partial x_{i}}\left(x^{\prime}\right)+\varepsilon x_{n} H_{i}^{k}\left(\varepsilon x^{\prime}\right) \frac{\partial \varphi_{\varepsilon}}{\partial x_{k}}\left(x^{\prime}\right), \frac{\partial \varphi_{\varepsilon}}{\partial x_{j}}\left(x^{\prime}\right)+\varepsilon x_{n} H_{j}^{l}\left(\varepsilon x^{\prime}\right) \frac{\partial \varphi_{\varepsilon}}{\partial x_{l}}\left(x^{\prime}\right)\right) \\
& =\bar{g}_{i j}+\varepsilon x_{n}\left(H_{i}^{k} \bar{g}_{k j}+H_{j}^{l} \bar{g}_{i l}\right)+\varepsilon^{2} x_{n}^{2} H_{i}^{k} H_{j}^{l} \bar{g}_{k l} .
\end{aligned}
$$

Note that also the inverse matrix $\left\{g^{A B}\right\}$ decomposes as

$$
g^{A B}=\left(\begin{array}{cc}
\left\{g^{i j}\right\} & 0 \\
0 & 1
\end{array}\right) .
$$

We begin by finding a first-order approximation for $u_{\varepsilon}$, to show the ideas of the general procedure. We define the following map $u \mapsto \tilde{u}$ from functions on $\partial \Omega_{\varepsilon}$ (resp. $\left.\partial \Omega_{\varepsilon} \times \mathbb{R}_{+}\right)$into functions on $\partial \Omega\left(\right.$ resp. $\left.\partial \Omega \times \mathbb{R}_{+}\right)$

$\tilde{u}\left(\varepsilon x^{\prime}\right)=u\left(x^{\prime}\right)\left(\operatorname{resp} . \tilde{u}\left(\varepsilon x^{\prime}, x_{n}\right)=u\left(x^{\prime}, x_{n}\right)\right), x^{\prime} \in \partial \Omega_{\varepsilon}\left(\operatorname{resp} .\left(x^{\prime}, x_{n}\right) \in \partial \Omega_{\varepsilon} \times \mathbb{R}_{+}\right)$.

Using the above parametrization, we look for an approximate solution $u_{1, \varepsilon}$ of the form

$$
u_{1, \varepsilon}\left(x^{\prime}, x_{n}\right)=w_{0}+\varepsilon \tilde{w}_{1}\left(\varepsilon x^{\prime}, x_{n}\right),
$$

where the function $\tilde{w}_{1}$ is defined on $\partial \Omega \times \mathbb{R}_{+}$, consistently with (22). We note that, from the above decomposition of $g_{A B}$ (and $g^{A B}$ ), one has

$$
\begin{aligned}
-\Delta_{g} u & =-g^{A B} u_{A B}-\frac{1}{\sqrt{\operatorname{det} g}} \partial_{A}\left(g^{A B} \sqrt{\operatorname{det} g}\right) u_{B} \\
& =-u_{n n}-g^{i j} u_{i j}-\frac{1}{\sqrt{\operatorname{det} g}} \partial_{n}(\sqrt{\operatorname{det} g}) u_{n}-\frac{1}{\sqrt{\operatorname{det} g}} \partial_{i}\left(g^{i j} \sqrt{\operatorname{det} g}\right) u_{j} .
\end{aligned}
$$

We have, formally

$$
\operatorname{det} g=\operatorname{det}\left(\bar{g}^{-1} g\right) \operatorname{det} \bar{g}=(\operatorname{det} \bar{g})\left(1+\varepsilon x_{n} \operatorname{tr}\left(\bar{g}^{-1} \alpha\right)\right)+o(\varepsilon),
$$

where

$$
\alpha_{i j}=H_{i}^{k} \bar{g}_{k j}+H_{j}^{l} \bar{g}_{i l}
$$


There holds

$$
\left(\bar{g}^{-1} \alpha\right)_{i s}=\bar{g}^{s j} \alpha_{i j}=\bar{g}^{s j}\left(H_{i}^{k} \bar{g}_{k j}+H_{j}^{l} \bar{g}_{i l}\right)
$$

and hence

$$
\operatorname{tr}\left(\bar{g}^{-1} \alpha\right)=\bar{g}^{i j}\left(H_{i}^{k} \bar{g}_{k j}+H_{j}^{l} \bar{g}_{i l}\right)=2 \bar{g}^{i j} H_{i}^{k} \bar{g}_{k j}=2 H_{i}^{i} .
$$

We recall that the quantity $H_{i}^{i}$ represents the opposite of the mean curvature of $\partial \Omega$ (by our choice of $\nu$ ), and in particular it is independent of the choice of coordinates.

Using (23), (24), writing formally $\left(w_{0}+\varepsilon w_{1}\right)^{p}$ as $w_{0}^{p}+p \varepsilon w_{0}^{p-1} w_{1}+o(\varepsilon)$, and expanding $-\Delta_{g_{\varepsilon}} u_{1, \varepsilon}+u_{1, \varepsilon}=u_{1, \varepsilon}^{p}$ up to the first order in $\varepsilon$, we obtain the following equation for $w_{1}$

$$
\left\{\begin{array}{l}
-w_{1}^{\prime \prime}+w_{1}-p w_{0}^{p-1} w_{1}=H_{1}^{1} w_{0}^{\prime} \quad \text { in } \mathbb{R}_{+}, \\
w_{1}^{\prime}(0)=0 .
\end{array}\right.
$$

By Proposition 2.1 and the subsequent comment there, equation (25) can be solved for any right-hand side in $L^{2}\left(\mathbb{R}_{+}^{2}\right)$, as in this case (see equation (5)). The resulting expression for $w_{1}$ will be independent of the choice of coordinates on $\partial \Omega_{\varepsilon}$.

The construction of a better approximate solution is performed by expanding formally the equation $-\Delta_{g_{\varepsilon}} u+u=u^{p}$ in powers of $\varepsilon$ up to order $k$. A rigorous proof is in [36].

For smooth functions $\tilde{w}_{1}, \ldots \tilde{w}_{k}: \partial \Omega \times \mathbb{R}_{+}$, let

$$
\hat{u}_{k, \varepsilon}\left(x^{\prime}, x_{n}\right)=w_{0}\left(x_{n}\right)+\varepsilon \tilde{w}_{1}\left(\varepsilon x^{\prime}, x_{n}\right)+\ldots \varepsilon^{k} \tilde{w}_{k}\left(\varepsilon x^{\prime}, x_{n}\right) ; \quad x^{\prime} \in \partial \Omega_{\varepsilon}, x_{n} \in \mathbb{R}_{+} .
$$

Expanding formally the equation $-\Delta_{g} u+u=u^{p}$ (imposing Neumann boundary conditions) in powers of $\varepsilon$, we find that $\tilde{w}_{i}$ satisfies the following recurrence 
formula for $i$ running from 1 to $k$

$$
\begin{cases}-\tilde{w}_{i}^{\prime \prime}+\tilde{w}_{i}-p w_{0}^{p-1} \tilde{w}_{i}=\sum_{j=1}^{i} x_{n}^{j-1} \tilde{G}_{i}\left(x^{\prime}\right) \tilde{w}_{i-j}^{\prime}\left(\varepsilon x^{\prime}, x_{n}\right)-\sum_{j=0}^{i-2}\left(L_{i-j-2} \tilde{w}_{j}\right)\left(\varepsilon x^{\prime}, x_{n}\right) \\ +\sum_{\substack{j_{1}, \ldots, j_{j-1} \\ \sum l j_{l}=i}} C_{i, j_{1}, \ldots, j_{i}} w_{0}^{p-\sum j_{l}} \tilde{w}_{1}^{j_{1}} \cdots \tilde{w}_{i-1}^{j_{i-1}}\left(\varepsilon x^{\prime}, x_{n}\right) ; & \text { in } \mathbb{R}_{+} \\ \tilde{w}_{k}^{\prime}(0)=0, & \end{cases}
$$

where the derivatives are taken with respect to the variable $x_{n}$, the coefficients $C_{i, j_{1}, \ldots, j_{i}}$ are constants, $\tilde{G}_{i}: \partial \Omega \rightarrow \mathbb{R}$ are smooth functions and $L_{i-j-2}$ are linear second-order differential operators.

Proceeding by induction and using standard estimates on solutions of ODE's, we find that there exist polynomials $P_{i}(t)$ such that

$$
\left\{\begin{array}{l}
\left\|\left(\nabla^{\prime}\right)^{(m)} \tilde{w}_{i}\left(x^{\prime}, x_{n}\right)\right\|^{\prime} \leq C_{i, m} P_{i}\left(x_{n}\right) e^{-x_{n}} ; \\
\left\|\left(\nabla^{\prime}\right)^{(m)} \tilde{w}_{i}^{\prime}\left(x^{\prime}, x_{n}\right)\right\|^{\prime} \leq C_{i, m} P_{i}\left(x_{n}\right) e^{-x_{n}} ; \\
\left\|\left(\nabla^{\prime}\right)^{(m)} \tilde{w}_{i}^{\prime \prime}\left(x^{\prime}, x_{n}\right)\right\|^{\prime} \leq C_{i, m} P_{i}\left(x_{n}\right) e^{-x_{n}} ;
\end{array} \quad x^{\prime} \in \partial \Omega_{\varepsilon}, x_{n} \in \mathbb{R}_{+}, m=0,1, \ldots,\right.
$$

where $\nabla^{\prime}$ denotes the derivative with respect to $x^{\prime}$, and $C_{i, m}$ is a constant depending only on $\Omega, p, i$ and $m$. In particular these decay estimates ensure that the right-hand side in (26) belongs to $L^{2}\left(\mathbb{R}_{+}\right)$, and (26) is solvable for all $i=1, \ldots, k$.

Therefore, $u_{k, \varepsilon}$ is obtained by multiplying $\hat{u}_{k, \varepsilon}$ by a cutoff function supported in $\left\{x_{n} \in\left[0, \varepsilon^{-\gamma}\right]\right\}$.

\subsection{Eigenvalues of $T_{\Sigma_{\varepsilon}}$}

Let $\Sigma_{\varepsilon}$ be defined as

$$
\Sigma_{\varepsilon}=\Gamma_{\varepsilon}\left(S_{\varepsilon}\right):=\Gamma_{\varepsilon}\left(\partial \Omega_{\varepsilon} \times J_{\varepsilon}\right)=\left\{x \in \mathbb{R}^{n}: \operatorname{dist}\left(x, \partial \Omega_{\varepsilon}\right)<\varepsilon^{-\gamma}\right\} .
$$


We endow $\Sigma_{\varepsilon}$ with the metric $g_{\varepsilon}$ induced by the inclusion in $\mathbb{R}^{n}$, and we define the functional space

$$
H_{\Sigma_{\varepsilon}}=\left\{u \in H^{1}\left(\Sigma_{\varepsilon}\right): u\left(\Gamma_{\varepsilon}\left(x^{\prime}, \varepsilon^{-\gamma}\right)\right)=0 \text { for all } x^{\prime} \in \partial \Omega_{\varepsilon}\right\},
$$

with its natural norm

$$
\|u\|_{H_{\Sigma_{\varepsilon}}}^{2}=\int_{\Sigma_{\varepsilon}}\left(\left|\nabla_{g_{\varepsilon}} u\right|^{2}+u^{2}\right) ; \quad u \in H_{\Sigma_{\varepsilon}}
$$

and the corresponding scalar product $(,)_{H_{\Sigma_{\varepsilon}}}$. Using the expression of the metric $g_{\varepsilon}$, see equation (21) and the subsequent formulas, one finds

$$
\left(1-C \varepsilon^{1-\gamma}\right)\|u\|_{H_{S_{\varepsilon}}} \leq\|u\|_{H_{\Sigma_{\varepsilon}}} \leq\left(1+C \varepsilon^{1-\gamma}\right)\|u\|_{H_{S_{\varepsilon}}} ; \quad \text { for all } u \in H_{S_{\varepsilon}},
$$

where $C$ is a constant depending only on $\Omega$.

Let $u_{k, \varepsilon}$ be the function constructed in Subsection 2.2. Then we define $T_{\Sigma_{\varepsilon}}: H_{\Sigma_{\varepsilon}} \rightarrow H_{\Sigma_{\varepsilon}}$ in the following way

$$
\left(T_{\Sigma_{\varepsilon}} u, v\right)_{H_{\Sigma_{\varepsilon}}}=\int_{\Sigma_{\varepsilon}}\left(\nabla_{g_{\varepsilon}} u \cdot \nabla_{g_{\varepsilon}} v+u v-p u_{k, \varepsilon}^{p-1} u v\right) d V_{g_{\varepsilon}} ; \quad u, v \in H_{\Sigma_{\varepsilon}} .
$$

The goal of this subsection is to study the eigenvalues $\sigma$ of the problem

$$
T_{\Sigma_{\varepsilon}} u=\sigma u, \quad u \in H_{\Sigma_{\varepsilon}} .
$$

In the next Proposition we characterize the eigenfunctions of $T_{\Sigma_{\varepsilon}}$ when the eigenvalue $\sigma$ is close to zero. We recall the definition of the functions $\phi_{l}$ and $v_{l, \varepsilon}$ from Subsection 2.1. Proposition 2.9 is the counterpart of Proposition 2.5 for a non-flat metric.

Proposition 2.9 Let $\gamma>0$ be sufficiently small, and let $\Lambda \in(0, \tau / 4)$. Suppose $\sigma \in[-\tau / 4, \tau / 4]$, and let $u$ be an eigenfunction of $T_{\Sigma_{\varepsilon}}$ with eigenvalue $\sigma$. Then there holds

$$
\left\|u-\sum_{\left\{l: \mu_{l, \varepsilon} \in[\sigma-\Lambda, \sigma+\Lambda]\right\}} \alpha_{l} \phi_{l} v_{l, \varepsilon}\right\|_{H_{\Sigma_{\varepsilon}}} \leq C \frac{\varepsilon^{1-\gamma}}{\Lambda}\|u\|_{H_{\Sigma_{\varepsilon}}},
$$

for some coefficients $\left\{\alpha_{l}\right\}$ and for some constant $C$ depending only on $\Omega$ and $p$. 
Let $\bar{u}_{k, \varepsilon}: \Omega \rightarrow \mathbb{R}$ be the function defined by

$$
\bar{u}_{k, \varepsilon}(x)=u_{k, \varepsilon}(\varepsilon x), \quad x \in \Omega .
$$

We recall the following result from [35].

Proposition 2.10 The eigenvalues of the operator $T_{\Sigma_{\varepsilon}}$ are differentiable with respect to $\varepsilon$, the eigenvalues being considered a possibly multivalued function of $\varepsilon$. If $\sigma(\varepsilon)$ is such an eigenvalue, then

$$
\frac{\partial \sigma}{\partial \varepsilon}=\left\{\text { eigenvalues of } Q_{\sigma}\right\},
$$

where $Q_{\sigma}: H_{\sigma} \times H_{\sigma} \rightarrow \mathbb{R}$ is the quadratic form given by

$$
Q_{\sigma}(u, v)=(1-\sigma) \frac{2}{\varepsilon} \int_{\Sigma_{\varepsilon}} \nabla u \cdot \nabla v-p(p-1) \int_{\Sigma_{\varepsilon}} u v u_{k, \varepsilon}^{p-2} \frac{\partial \bar{u}_{k, \varepsilon}}{\partial \varepsilon}(\varepsilon \cdot) .
$$

Here $H_{\sigma} \subseteq H_{\Sigma_{\varepsilon}}$ denotes the eigenspace of $T_{\Sigma_{\varepsilon}}$ corresponding to $\sigma$ and the function $u_{k, \varepsilon}$ is defined in Subsection 2.2.

We apply Proposition 2.10 to the eigenvalues $\sigma$ which are close to 0 .

Proposition 2.11 Let $\sigma$ be an eigenvalue of $T_{\Sigma_{\varepsilon}}$ belonging to the interval $[-\tau / 4, \tau / 4]$. Then there holds

$$
\left|\frac{\partial \sigma}{\partial \varepsilon}-\frac{1}{\varepsilon} \tilde{F}(\sigma)\right| \leq C \varepsilon^{-\frac{1+\gamma}{2}},
$$

where $\gamma$ is sufficiently small, $\tilde{F}(\cdot)$ is given in $(10)$, and $C$ is a constant depending only on $\Omega$ and $p$.

Now we are in position to prove the following proposition, which characterizes the spectrum of $T_{\Sigma_{\varepsilon}}$.

Proposition 2.12 Let $u_{k, \varepsilon}$ and $T_{\Sigma_{\varepsilon}}$ be as above. Then there exists a positive constant $C$, depending on $p, \Omega$ and $k$ with the following property. For a suitable sequence $\varepsilon_{j} \rightarrow 0$, the operator $T_{\Sigma_{\varepsilon_{j}}}: H_{\Sigma_{\varepsilon_{j}}} \rightarrow H_{\Sigma_{\varepsilon_{j}}}$ is invertible and the inverse operator satisfies

$$
\left\|T_{\Sigma_{\varepsilon_{j}}}^{-1}\right\| \leq \frac{C}{\varepsilon_{j}^{n-1}}, \quad \text { for all } j \in \mathbb{N}
$$


Proof. Let $\left\{\sigma_{j}\right\}_{j}$ (resp. $\left\{s_{j}\right\}_{j}$ ) denote the eigenvalues of $T_{\Sigma_{\varepsilon}}$ (resp. $T_{S_{\varepsilon}}$ ), counted in increasing order with their multiplicity. Let also

$$
\left.N\left(\Sigma_{\varepsilon}\right)=\sharp\left\{j: \sigma_{j} \leq 0\right\} ; \quad \text { (resp. } N\left(S_{\varepsilon}\right)=\sharp\left\{j: s_{j} \leq 0\right\}\right) .
$$

denote the number of non-positive eigenvalues of $T_{\Sigma_{\varepsilon}}$ (resp. $T_{S_{\varepsilon}}$ ). Recall that, from Proposition 2.5, the eigenvalues of $T_{S_{\varepsilon}}$ coincide with the numbers $\left\{\mu_{j, \varepsilon}\right\}_{j}:=$ $\mu_{\varepsilon^{2} \lambda_{j}, \varepsilon}$, where $\left\{\lambda_{j}\right\}_{j}$ are the eigenvalues of the Laplace-Beltrami operator $-\Delta_{\hat{g}}$, see also equations (12), (15). We also recall the Weyl's asymptotic formula, see e.g. [13] page 169

$$
\lambda_{j} \sim \frac{(2 \pi)^{2}}{\omega_{n-1}^{\frac{2}{n-1}}}\left(\frac{j}{\operatorname{Vol}(\partial \Omega)}\right)^{\frac{2}{n-1}}=C_{n, \Omega} j^{\frac{2}{n-1}}, \quad \text { as } j \rightarrow+\infty .
$$

From the strict monotonicity of $\alpha \mapsto \mu_{\alpha}:=\mu(\alpha)$ and from the last formula we deduce

$$
\sharp\left\{j: \varepsilon^{2} \lambda_{j} \leq \mu^{-1}(0)\right\} \sim \tilde{C}_{n, \Omega, p} \varepsilon^{1-n} .
$$

From Proposition 2.3, we have

$$
\left|\mu_{\varepsilon^{2} \lambda_{j}, \varepsilon}-\mu_{\varepsilon^{2} \lambda_{j}}\right| \leq C e^{-\delta \varepsilon^{-\gamma}} ; \quad \text { provided } \mu_{\varepsilon^{2} \lambda_{j}} \in\left[-\frac{\tau}{4}, \frac{\tau}{4}\right],
$$

which implies

$\sharp\left\{j: \varepsilon^{2} \lambda_{j} \leq \mu^{-1}(0)-C e^{-\delta \varepsilon^{-\gamma}}\right\} \leq N\left(S_{\varepsilon}\right) \leq \sharp\left\{j: \varepsilon^{2} \lambda_{j} \leq \mu^{-1}(0)+C e^{-\delta \varepsilon^{-\gamma}}\right\}$

and hence

$$
N\left(S_{\varepsilon}\right) \sim \tilde{C}_{n, \Omega, p} \varepsilon^{1-n} .
$$

From the Courant-Fischer method, we get

$$
\sigma_{j}=\inf \left\{\sup _{u \in M} \frac{\left(T_{\Sigma_{\varepsilon}} u, u\right)_{H_{\Sigma_{\varepsilon}}}}{\|u\|_{H_{\Sigma_{\varepsilon}}}^{2}}: M \text { subspace of } H_{\Sigma_{\varepsilon}}, \operatorname{dim} M=j\right\}
$$

and a similar characterization for $s_{j}$. There holds

$$
\begin{aligned}
& \left|\left(T_{\Sigma_{\varepsilon}} u, u\right)_{H_{\Sigma_{\varepsilon}}}-\left(T_{S_{\varepsilon}} u, u\right)_{H_{S_{\varepsilon}}}\right| \leq C \varepsilon^{1-\gamma}\|u\|_{H_{S_{\varepsilon}}}^{2} \\
& \left|\|u\|_{H_{\Sigma_{\varepsilon}}}^{2}-\|u\|_{H_{S_{\varepsilon}}}^{2}\right| \leq C \varepsilon^{1-\gamma}\|u\|_{H_{S_{\varepsilon}}}^{2},
\end{aligned}
$$


for all $u \in H_{\Sigma_{\varepsilon}}$ and for some constant $C$ depending only on $\Omega$ and $p$. Note that, by $(28)$, in the last inequality we can also substitute $\|\cdot\|_{H_{S_{\varepsilon}}}$ with $\|\cdot\|_{H_{\Sigma_{\varepsilon}}}$. The last two formulas yield

$$
\left|s_{j}-\sigma_{j}\right| \leq C \varepsilon^{1-\gamma}, \quad \text { for all } j,
$$

where $C$ depends only on $\Omega$ and $p$. From (36), (38) there exists a positive constant $C$, depending only on $\Omega$ and $p$, such that

$$
\sharp\left\{j: \varepsilon^{2} \lambda_{j} \leq \mu^{-1}(0)-C \varepsilon^{1-\gamma}\right\} \leq N\left(\Sigma_{\varepsilon}\right) \leq \sharp\left\{j: \varepsilon^{2} \lambda_{j} \leq \mu^{-1}(0)+C \varepsilon^{1-\gamma}\right\},
$$

which implies

$$
N\left(\Sigma_{\varepsilon}\right) \sim \sharp\left\{j: \lambda_{j} \leq \frac{\mu^{-1}(0)}{\varepsilon^{2}}\right\} \sim\left(\frac{\mu^{-1}(0)}{\varepsilon^{2} C_{n, \Omega}}\right)^{\frac{n-1}{2}}=\tilde{C}_{n, \Omega, p} \varepsilon^{1-n} .
$$

For $l \in \mathbb{N}$, let $\varepsilon_{l}=2^{-l}$. Then from (39) we have

$$
N\left(\Sigma_{\varepsilon_{l+1}}\right)-N\left(\Sigma_{\varepsilon_{l}}\right) \sim \tilde{C}_{n, \Omega, p}\left(2^{(l+1)(n-1)}-2^{l(n-1)}\right)=\tilde{C}_{n, \Omega, p}\left(2^{n-1}-1\right) \varepsilon_{l}^{1-n} .
$$

Note that, by Proposition 2.11, the eigenvalues of $T_{\Sigma_{\varepsilon}}$ close to 0 decrease when $\varepsilon$ decreases to zero. In other words, by the last equation, the number of eigenvalues which cross 0 , when $\varepsilon$ decreases from $\varepsilon_{l}$ to $\varepsilon_{l+1}$, is of order $\varepsilon_{l}^{1-n}$. Now we define

$$
A_{l}=\left\{\varepsilon \in\left(\varepsilon_{l+1}, \varepsilon_{l}\right): \operatorname{ker} T_{\Sigma_{\varepsilon}} \neq \emptyset\right\} ; \quad B_{l}=\left(\varepsilon_{l+1}, \varepsilon_{l}\right) \backslash A_{l} .
$$

By Proposition 2.11 and (40), it follows that card $\left(A_{l}\right)<C \varepsilon_{l}^{1-n}$, and hence there exists an interval $\left(a_{l}, b_{l}\right)$ such that

$$
\left(a_{l}, b_{l}\right) \subseteq B_{l} ; \quad\left|b_{l}-a_{l}\right| \geq C^{-1} \frac{\operatorname{meas}\left(B_{l}\right)}{\operatorname{card}\left(A_{l}\right)} \geq C^{-1} \varepsilon_{l}^{n} .
$$

From Proposition 2.11 we deduce that

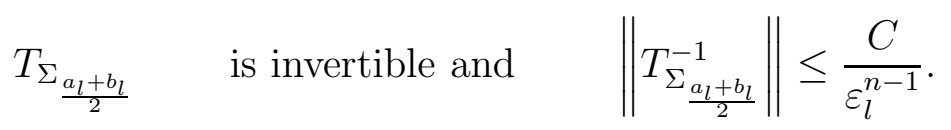

Now it is sufficient to set $\varepsilon_{j}=\frac{a_{j}+b_{j}}{2}$. This concludes the proof. 


\subsection{Proof of Theorem 1.1}

In this subsection we prove Theorem 1.1. We just treat the case $p \leq \frac{n+2}{n-2}$. For $p>\frac{n+2}{n-2}$, see [36].

The following result can be proved as is [35], Proposition 5.6.

Proposition 2.13 Let $k \in \mathbb{N}$ be fixed, and let $u_{k, \varepsilon}$ be as in Subsection 2.2. Let $\left\{\sigma_{j}\right\}_{j}$ denote the eigenvalues of $T_{\Sigma_{\varepsilon}}$, counted in increasing order and with their multiplicity, and let $\left\{\hat{\sigma}_{j}\right\}_{j}$ denote the eigenvalues of $I_{\varepsilon}^{\prime \prime}\left(u_{k, \varepsilon}\right)$. Then there exists a positive constant $C$, depending only on $\Omega$ and $p$, such that

$$
\left|\sigma_{j}-\hat{\sigma}_{j}\right| \leq C e^{-\delta \varepsilon^{-\frac{\gamma}{2}}} ; \quad \text { whenever } \sigma_{j} \in\left[-\frac{\tau}{4}, \frac{\tau}{4}\right]
$$

The proof of this proposition consists in showing that the eigenfunctions of $I_{\varepsilon}^{\prime \prime}\left(u_{k, \varepsilon}\right)$ decay exponentially away from $\partial \Omega_{\varepsilon}$, and multiplying by suitable cut-off functions. The eigenvalues $\sigma_{j}$ and $\hat{\sigma}_{j}$ are then compared using the CourantFisher method, as in the proof of Proposition 2.3.

Let $\varepsilon_{j}$ be as in Proposition 2.12. Then from Proposition 2.13 we deduce

$$
I_{\varepsilon_{j}}^{\prime \prime}\left(u_{k, \varepsilon_{j}}\right) \quad \text { is invertible and } \quad\left\|I_{\varepsilon_{j}}^{\prime \prime}\left(u_{k, \varepsilon_{j}}\right)^{-1}\right\| \leq \frac{C}{\varepsilon_{j}^{n-1}} .
$$

For brevity, in the rest of the proof, we simply write $\varepsilon$ instead of $\varepsilon_{j}$. We apply the contraction mapping theorem, looking for a solution $u_{\varepsilon}$ of the form

$$
u_{\varepsilon}=u_{k, \varepsilon}+w, \quad w \in H^{1}\left(\Omega_{\varepsilon}\right) .
$$

If $I_{\varepsilon}^{\prime \prime}\left(u_{k, \varepsilon}\right)$ is invertible (which is true along the sequence $\varepsilon_{j}$ ), we can write

$$
I_{\varepsilon}^{\prime \prime}\left(u_{k, \varepsilon}+w\right)=0 \quad \Leftrightarrow \quad w=-\left(I_{\varepsilon}^{\prime \prime}\left(u_{k, \varepsilon}\right)\right)^{-1}\left[I_{\varepsilon}^{\prime}\left(u_{k, \varepsilon}\right)+G(w)\right]
$$

where

$$
G(w)=I_{\varepsilon}^{\prime}\left(u_{k, \varepsilon}+w\right)-I_{\varepsilon}^{\prime}\left(u_{k, \varepsilon}\right)-I_{\varepsilon}^{\prime \prime}\left(u_{k, \varepsilon}\right)[w] .
$$

Let us define the operator $F_{\varepsilon}: H^{1}\left(\Omega_{\varepsilon}\right) \rightarrow H^{1}\left(\Omega_{\varepsilon}\right)$

$$
F_{\varepsilon}(w)=-\left(I_{\varepsilon}^{\prime \prime}\left(u_{k, \varepsilon}\right)\right)^{-1}\left[I_{\varepsilon}^{\prime}\left(u_{k, \varepsilon}\right)+G(w)\right], \quad w \in H^{1}\left(\Omega_{\varepsilon}\right) .
$$


Let $\varepsilon_{j}$ be given by Proposition 2.12. We are going to prove that $F_{\varepsilon}$ is a contraction in a suitable closed set of $H^{1}\left(\Omega_{\varepsilon}\right)$. From Proposition 2.8 and (42) we get

$$
\begin{aligned}
& \left\|F_{\varepsilon_{j}}(w)\right\| \leq\left\{\begin{array}{ll}
C \varepsilon_{j}^{-(n-1)}\left(\varepsilon_{j}^{k+1-\frac{n-1}{2}}+\|w\|^{p}\right) & \text { for } p \leq 2, \\
C \varepsilon_{j}^{-(n-1)}\left(\varepsilon_{j}^{k+1-\frac{n-1}{2}}+\|w\|^{2}\right) & \text { for } p>2 ;
\end{array} \quad\|w\| \leq 1\right. \\
& \left\|F_{\varepsilon_{j}}\left(w_{1}\right)-F_{\varepsilon_{j}}\left(w_{2}\right)\right\| \leq\left\{\begin{array}{cc}
C \varepsilon_{j}^{-(n-1)}\left(\left\|w_{1}\right\|^{p-1}+\left\|w_{2}\right\|^{p-1}\right)\left\|w_{1}-w_{2}\right\| & p \leq 2 \\
\left\|w_{1}\right\|,\left\|w_{2}\right\| \leq 1 . & \\
C \varepsilon_{j}^{-(n-1)}\left(\left\|w_{1}\right\|+\left\|w_{2}\right\|\right)\left\|w_{1}-w_{2}\right\| & p>2
\end{array}\right.
\end{aligned}
$$

Now we choose integers $d$ and $k$ such that

$$
d>\left\{\begin{array}{ll}
\frac{n-1}{p-1} & \text { for } p \leq 2, \\
n-1 & \text { for } p>2
\end{array} \quad k+1>d+\frac{3}{2}(n-1)\right.
$$

and we set

$$
\mathcal{B}_{j}=\left\{w \in H^{1}\left(\Omega_{\varepsilon}\right):\|w\| \leq \varepsilon_{j}^{d}\right\}
$$

From (44), (45) it follows that $F_{\varepsilon_{j}}$ is a contraction in $\mathcal{B}_{j}$ for $\varepsilon_{j}$ small. Since $u_{\varepsilon}$ is close in norm to a positive function $\left(u_{k, \varepsilon}\right)$, the positivity of $u_{\varepsilon}$ follows from standard arguments. The points i) and ii) in the statement follow from the construction of $u_{\varepsilon}$. This concludes the proof.

Remark 2.14 By the above construction and some standard estimates, there holds

$$
I_{\varepsilon_{j}}^{\prime \prime}\left(u_{\varepsilon_{j}}\right)=I_{\varepsilon_{j}}^{\prime \prime}\left(u_{k, \varepsilon_{j}}\right)+O\left(\varepsilon^{d \min \{1, p-1\}}\right)=I_{\varepsilon_{j}}^{\prime \prime}\left(u_{k, \varepsilon_{j}}\right)+o\left(\varepsilon_{j}^{n}\right) .
$$

It follows from Propositions 2.12, 2.13 that the Morse index of $u_{\varepsilon_{j}}$ coincides with $N\left(\Sigma_{\varepsilon_{j}}\right) \sim \varepsilon_{j}^{1-n}$. 


\section{References}

[1] Agmon, S., Douglis, A., Nirenberg, L., Estimates near the boundary for solutions of elliptic partial differential equations satisfying general boundary conditions, I. Comm. Pure Appl. Math. 12 (1959), 623-727.

[2] Ambrosetti A.; Badiale M., Homoclinics: Poincaré-Melnikov type results via a variational approach, Ann. Inst. Henri. Poincaré Analyse Non Linéaire 15 (1998), 233-252.

[3] Ambrosetti, A.; Badiale, M.; Cingolani, S., Semiclassical states of nonlinear Schrodinger equations, Arch. Rational Mech. Anal. 140 (1997), 285-300.

[4] Ambrosetti, A.; Malchiodi, A.; Ni, W.M., Singularly Perturbed Elliptic Equations with Symmetry: Existence of Solutions Concentrating on Spheres, Part I, Comm. Math. Phys. 235 (2003), 427-466.

[5] Ambrosetti, A.; Malchiodi, A.; Ni, W.M., Singularly Perturbed Elliptic Equations with Symmetry: Existence of Solutions Concentrating on Spheres, Part II, Indiana Univ. Math. J. 53, No. 2, (2004), 297-329.

[6] Ambrosetti, A.; Malchiodi, A.; Ni, W.M., Solutions, concentrating on spheres, to symmetric singularly perturbed problems, C. R. Math. Acad. Sci. Paris 335, No. 2, (2002), 145-150.

[7] Ambrosetti, A.; Malchiodi, A.; Secchi, S., Multiplicity results for some nonlinear singularly perturbed elliptic problems on $R^{n}$, Arch. Rat. Mech. Anal. 159, 3 (2001) 253-271.

[8] Badiale, M.; D'Aprile, T., Concentration around a sphere for a singularly perturbed Schrdinger equation, Nonlinear Anal. 49, no. 7, Ser. A: Theory Methods,(2002), 947-985.

[9] Ben Ayed, M.; El Mehdi, K.; Hammami, M., A nonexistence result for Yamabe type problems on thin annuli, Ann. Inst. H. Poincar Anal. Non Linaire 19, No. 5, (2002), 715-744. 
[10] Benci, V.; D'Aprile, T., The semiclassical limit of the nonlinear Schrödinger equation in a radial potential, J. Differential Equations 184, No. 1, (2002), 109-138.

[11] Brendle, S., On Solutions to the Ginzburg-Landau equations in higher dimensions, preprint, (2002).

[12] Brendle, S., On the construction of solutions of the Yang-Mills equations in higher dimensions, preprint, (2002).

[13] Chavel, I., Riemannian geometry, a modern introduction, Cambridge Tracts in Mathematics, 108. Cambridge University Press, Cambridge, (1993), xii+386.

[14] Cingolani, S.; Pistoia, A., Nonexistence of single blow-up solutions for a nonlinear Schrödinger equation involving critical Sobolev exponent, Z. Angew. Math. Phys. 55, no. 2, (2004), 201-215.

[15] Dancer, E.N., New solutions of equations on $\mathbb{R}^{n}$, Ann. Scuola Norm. Sup. Pisa Cl. Sci. (4) 30 (2001), No. 3-4, (2002), 535-563.

[16] Dancer, E.N., Stable and finite Morse index solutions on $R^{n}$ or on bounded domains with small diffusion, II. Indiana Univ. Math. J. 53, No. 1, (2004), 97-108.

[17] Dancer, E. N.; Yan, S., Multipeak solutions for a singularly perturbed Neumann problem, Pacific J. Math. 189, No. 2, (1999), 241-262.

[18] D'Aprile T., On a class of solutions with non vanishing angular momentum for nonlinear Schroedinger equation, Diff. Int. Eq. 16, No. 3, (2003), 349384.

[19] Del Pino, M.; Felmer, P.; Wei, J., On the role of the mean curvature in some singularly perturbed Neumann problems, S.I.A.M. J. Math. Anal. 31 (1999), 63-79. 
[20] Del Pino, M.; Felmer, P.; Kowalczyk, M., Boundary spikes in the GiererMeinhardt system, Commun. Pure Appl. Anal. 1, No. 4, (2002), 437-456.

[21] Floer, A.; Weinstein, A., Nonspreading wave packets for the cubic Schrodinger equation with a bounded potential, J. Funct. Anal. 69 (1986), 397-408.

[22] Gierer, A.; Meinhardt, H., A theory of biological pattern formation, Kybernetik (Berlin), 12 (1972), 30-39.

[23] Gilbarg, D.; Trudinger, N.S., Elliptic partial differential equations of second order, Grundlehren der Mathematischen Wissenschaften, Vol. 224. Springer-Verlag, Berlin-New York, (1977) x+401.

[24] Grossi, M.; Pistoia, A.; Wei, J., Existence of multipeak solutions for a semilinear Neumann problem via nonsmooth critical point theory, Calc. Var. Partial Differential Equations 11, No. 2, (2000), 143-175.

[25] Gui, C., Multipeak solutions for a semilinear Neumann problem, Duke Math. J., 84 (1996), 739-769.

[26] Gui, C.; Wei, J., On multiple mixed interior and boundary peak solutions for some singularly perturbed Neumann problems, Canad. J. Math. 52, No. 3, (2000), 522-538.

[27] Gui, C.; Wei, J.; Winter, M., Multiple boundary peak solutions for some singularly perturbed Neumann problems, Ann. Inst. H. Poincar Anal. Non Linaire 17, No. 1, (2000), 47-82.

[28] Iron, D.; Ward, M.J.; Wei, J., The stability of spike solutions to the onedimensional Gierer-Meinhardt model, Phys. D 150, No. 1-2, (2001), 25-62.

[29] Kato, T., Perturbation theory for linear operators. Second edition, Grundlehren der Mathematischen Wissenschaften, Band 132. SpringerVerlag, Berlin-New York, (1976), xxi+619. 
[30] Li, Y. Y., On a singularly perturbed equation with Neumann boundary conditions, Comm. Partial Differential Equations 23 (1998), 487-545.

[31] Li, Y.Y., Nirenberg, L., The Dirichlet problem for singularly perturbed elliptic equations, Comm. Pure Appl. Math. 51 (1998), 1445-1490.

[32] Lin, C.S.; Ni, W.-M.; Takagi, I., Large amplitude stationary solutions to a chemotaxis systems, J. Differential Equations, 72 (1988), 1-27.

[33] Mahmoudi, F.; Mazzeo, R.; Pacard, F., Constant mean curvature hypersurfaces condensing along a submanifold, (preprint)

[34] Malchiodi, A., Adiabatic limits for some Newtonian systems in $R^{n}$, Asympt. Anal. 25 (2001), 149-181.

[35] Malchiodi, A.; Montenegro, M., Boundary concentration phenomena for a singularly perturbed elliptic problem, Comm. Pure Appl. Math. 55, No. 12, (2002), 1507-1568.

[36] Malchiodi, A.; Montenegro, M., Multidimensional Boundary-layers for a singularly perturbed Neumann problem, Duke Math. J. 124, No. 1, (2004), $105-143$.

[37] Mazzeo, R.; Pacard, F., Foliations by constant mean curvature tubes, (preprint)

[38] Ni, W. M., Diffusion, cross-diffusion, and their spike-layer steady states, Notices Amer. Math. Soc. 45, No. 1, (1998), 9-18.

[39] Ni, W.M.; Takagi, I., On the shape of least-energy solution to a semilinear Neumann problem, Comm. Pure Appl. Math., 41 (1991), 819-851.

[40] Ni, W.M.; Takagi, I., Locating the peaks of least-energy solutions to a semilinear Neumann problem, Duke Math. J. 70, (1993), 247-281. 
[41] Ni, W.M.; Takagi, I.; Yanagida, E., Stability of least energy patterns of the shadow system for an activator-inhibitor model, Recent topics in mathematics moving toward science and engineering, Japan J. Indust. Appl. Math. 18, No. 2, (2001), 259-272.

[42] Oh, Y.G., On positive mupltibum states of nonlinear Schrödinger equation under multpile well potentials, Comm. Math. Phys. 131 (1990), 223-253.

[43] Pacard, F., Ritoré M., From constant mean curvature hypersurfaces to the gradient theory of phase transitions, J. Differential Geom. 64, No. 3, (2003), 359-423.

[44] Shatah, J.; Zeng, C., Periodic solutions for Hamiltonian systems under strong constraining forces, J. Differential Equations 186, No. 2, (2002), 572-585.

[45] Shi, J., Semilinear Neumann boundary value problems on a rectangle Trans. Amer. Math. Soc. 354, No. 8, (2002), 3117-3154.

[46] Turing, A.M., The chemical basis of morphogenesis, Phil. Trans. Royal Soc. London, Series B, Biological Sciences, 237 (1952), 37-72.

[47] Wang, Z.Q., On the existence of multiple, single-peaked solutions for a semilinear Neumann problem, Arch. Rational Mech. Anal., 120 (1992), 375-399.

[48] Wei, J., On the boundary spike layer solutions of a singularly perturbed semilinear Neumann problem, J. Differential Equations, 134 (1997), 104133.

Scuola Internazionale Superiore

di Studi Avanzati

via Beirut 4

34014 Trieste, Italy

E-mail: malchiod@sissa.edu
Universidade Estadual de Campinas

IMECC, Departamento de Matematica Caixa Postal 6065 13083-970, Campinas, SP, Brasil

E-mail: msm@ime.unicamp.br 